\title{
CAMINHOS E DESAFIOS DE INOVAÇÕES E ORGANIZAÇÕES RUMO À SUSTENTABILIDADE PATHWAYS AND CHALLENGES OF INNOVATIONS AND ORGANIZATIONS TOWARDS SUSTAINABILITY
}

\author{
Diego de Queiroz Machado ${ }^{1}$ \\ Fátima Regina Ney Matos ${ }^{2}$ \\ Mário Henrique Ogasavara ${ }^{3}$ \\ Ana Silvia Rocha Ipiranga ${ }^{4}$
}

\section{RESUMO}

Este ensaio tem como objetivo analisar os caminhos e desafios de inovações e organizações rumo à sustentabilidade. Para tanto, parte-se da discussão dos principais paradigmas tecnológicos, com destaque para o paradigma da sustentabilidade, e duas correntes de pensamento relacionadas: o otimismo tecnológico e o paradoxo de Jevons. Posteriormente, são apresentadas características relativas às chamadas inovações sustentáveis e organizações inovadoras sustentáveis. Conclui-se pela necessidade de aprofundamento e apreciação de elementos ainda pouco explorados neste campo, como as características de inovações advindas do paradigma sustentável (produtividade radical de recursos, biomimética, química verde, ecologia industrial, energias renováveis e nanotecnologia verde), as trajetórias de desenvolvimento de inovações sustentáveis ou de aprendizagem em organizações inovadoras e sustentáveis.

Palavras-chave: Inovação. Novas Tecnologias. Sustentabilidade.

Manuscript first received/Recebido em 22/07/2018 Manuscript accepted/Aprovado em: 02/09/2019

${ }^{1}$ Professor adjunto na Faculdade de Economia, Administração, Atuária, Contabilidade e Secretariado Executivo (FEAAC) e no Programa de Pós-Graduação em Administração e Controladoria (PPAC) da Universidade Federal do Ceará (UFC). Doutor em Administração deEmpresas pela UniversidadedeFortaleza (UNIFOR). E-mail: diegoqueirozm@yahoo.com.br

2 Professora auxiliar do Instituto Superior M iguel Torga (ISM T), em Coimbra, e professora titular da Universidade Potiguar (UnP). Doutora em A dministração pela Universidade Federal dePernambuco (UFPE). E-mail: fneymatos@globo.com

3 Coordenador do Programa de M estrado e D outorado em Gestão Internacional (PM D GI) da Escola Superior de Propaganda e M arketing (ESPM -SP). Ph.D. em M anagement pela University of Tsukuba (Japão), Pós-doutorado na National University of Singapore (Cingapura). E-mail: mario.ogasavara@espm.br

${ }_{4}$ Professora adjunta da Universidade Estadual do Ceará (UECE). Doutora em Psicologia do Trabalho e da Organização pela Università Alma M ater Studiorum di Bologna, na Itália, e Pós-D outora em Administração pela EBAPE-FVG. E-mail: anasilviaipi@uol.com.br 


\section{ABSTRACT}

This essay aims to analyze the pathways and challenges of innovations and organizations towards sustainability. Therefore, it starts with the discussion of the main technological paradigms, with emphasis on the paradigm of sustainability, and two related currents of thought: technological optimism and the Jevons paradox. Subsequently, features related to so-called sustainable innovations and sustainable innovative organizations are presented. It is concluded that there is a need for deepening and appreciation of elements that have not yet been explored in this field, such as the characteristics of innovations coming from the sustainable paradigm (radical resource productivity, biomimetics, green chemistry, industrial ecology, renewable energy and green nanotechnology), the trajectories of development of sustainable innovations or learning in innovative and sustainable organizations.

Keywords: Innovation. New Technologies. Sustainability.

\section{INTRODUÇÃO}

Em busca de novos caminhos para o alcance da sustentabilidade, pesquisadores das mais variadas ciências têm buscado contribuir com a produção de teorias e conhecimentos a partir de movimentos de comunicação entre as diversas áreas, como a administração, a sociologia, a biologia e a física. Inicialmente tendo ganhado força na década de 1980 com pesquisas voltadas principalmente para o âmbito da agricultura e preservação ambiental, as pesquisas em sustentabilidade já se expandiram para os mais diversos campos científicos, explorando questões abrangentes, como as dimensões políticas dos modelos de sustentabilidade (DIAS; SEIXAS, 2018), geração de energia (JACINTO et al., 2017), produção de alimentos (GIL et al., 2019) e saúde pública (LHERMIE et al., 2019).

Em uma perspectiva mais voltada para o âmbito da gestão, um grande volume de pesquisas tem se voltado para a sustentabilidade no contexto das organizações contemporâneas, explorando aspectos da sustentabilidade organizacional. A fim de favorecer uma inclusão maior de organizações de todos os tipos no campo da sustentabilidade, estas pesquisas focam na análise de elementos específicos da gestão das empresas, como os sistemas de informação (ANTHONY JR., 2019), ferramentas de marketing (DUMITRIU et al., 2019), produção e gestão de materiais (FALQI; AHMED; MALLICK, 2019), desenvolvimento de novos produtos (KALISH et al., 2018) e parques tecnológicos (TORRES; ALVEZ; VIDAL, 2017). Neste contexto, a gestão da inovação é apontada como a principal ferramenta de aderência das organizações ao campo da sustentabilidade, sendo responsável pelo desenvolvimento de competências para lidar com novas tecnologias, novos mercados e novas condições ambientais ou regulamentares (SEEBODE; JEANRENAUD; BESSANT, 2012).

Desde os primeiros anos do século XXI, denota-se um crescente interesse em pesquisas que visem à admissão de elementos da sustentabilidade organizacional na gestão de inovações. Criticando a visão generalizada de que a inovação por si só conduz ao progresso social, Vollenbroek (2002) defende a inclusão da sustentabilidade, mais precisamente das suas dimensões econômica, social e ambiental, nesse contexto, de forma que a inovação seja conduzida para a sustentabilidade. Esse tipo de inovação com foco na sustentabilidade, segundo Hall e Vredenburg (2003), tem como principais características a incorporação das dimensões sociais e ambientais da sustentabilidade, além da preocupação com as gerações futuras. 
Mais recentemente e já considerando também as dimensões econômica, social e ambiental da sustentabilidade, Hansen, Grosse-Dunker e Reichwald (2009) desenvolveram o chamado sustainability innovation cube, o qual relaciona essas dimensões com diferentes tipos de inovações e estágios de ciclos de vida. Destacam-se também o estudo de Castiaux (2012), que analisou as novas capacidades de inovação necessárias para integrar os aspectos ambientais, sociais e econômicos da sustentabilidade organizacional, a pesquisa de Lazzarotti et al. (2014), os quais verificaram o impacto das tecnologias ambientais no desempenho econômico-financeiro de uma empresa, e o trabalho de Wicki e Hansen (2019), que exploraram o uso das inovações verdes pelas empresas na diversificação de seus mercados.

Considerando, portanto, o aumento considerável do interesse de pesquisa nesta área, demandamse esforços de compreensão e aprofundamento dos elementos que lhes são relativos, principalmente visando ao seu desenvolvimento consciente. Assim, este ensaio tem como objetivo analisar os caminhos e desafios de inovações e organizações rumo à sustentabilidade.

Para tanto, a fim de possibilitar uma compreensão do desenvolvimento de novas tecnologias dentro do pensamento sustentável, parte-se da discussão dos principais paradigmas tecnológicos, com destaque para o paradigma da sustentabilidade. Posteriormente, são discutidas duas correntes de pensamento relacionadas - o otimismo tecnológico e o paradoxo de Jevons, ambas influenciadoras do atual estágio de convergência entre inovação e sustentabilidade. Finalmente, com foco no contexto mais específico das organizações, são apresentadas características relativas às chamadas inovações sustentáveis e organizações inovadoras sustentáveis, com destaque para a sua importância em vista da almejada sustentabilidade.

\section{PARADIGMAS TECNOLÓGICOS E SUSTENTABILIDADE}

Paradigmas tecnológicos são definidos por Dosi $(1982,1988)$ como um padrão para a solução dos problemas da sociedade, sendo baseados em princípios derivados das ciências naturais e regras específicas, que buscam a aquisição e proteção de conhecimentos, definindo as necessidades que se propõe a satisfazer, os princípios científicos e a tecnologia utilizados nesta tarefa. Dessa forma, o desenvolvimento de novas tecnologias se dá dentro de diferentes paradigmas tecnológicos, chamados de paradigmas tecnoeconômicos ou revoluções tecnológicas (techno-economic paradigms, technologies revolutions) (FREEMAN; PEREZ, 1988).

Segundo Lavarello (2014), os paradigmas tecnológicos começam a surgir a partir da existência de várias oportunidades econômicas que, ao longo do seu desenvolvimento, têm um conjunto limitado de padrões de solução de problemas e base de conhecimento escolhidos. Dentro desses paradigmas tecnológicos, o processo de inovação se dá de forma bastante seletiva, focalizando direções precisas e acumulativas para a aquisição de capacidades na solução de problemas, culminando em padrões relativamente ordenados de inovação em cada uma dessas ondas: "Além disso, cada paradigma envolve modos específicos de busca, de bases de conhecimento e de combinações entre as formas privadas e públicas de conhecimento tecnológico" (DOSI, 1988, p. 118, tradução nossa). Essas revoluções envolvem profundas e longas mudanças, não apenas tecnológicas, mas também econômicas e sociais, levando a benefícios de longo prazo. Assim, eles acabam por proporcionar um período de crescimento econômico bastante significativo, juntamente com um processo de reestruturação social, até que uma nova onda seja formada (SEEBODE; JEANRENAUD; BESSANT, 2012). 
Como exposto por Hargroves e Smith (2013), desde a revolução industrial na Inglaterra, houve pelo menos cinco ondas de inovação que proporcionaram o desenvolvimento econômico. No final do século XVIII, a mecanização moderna permitiu o advento da indústria têxtil e do comércio. Posteriormente, as melhorias incorporadas à máquina a vapor permitiram o crescimento das indústrias, fornecendo uma imensa quantidade de postos de trabalho para a população. Já na primeira metade do século XX, as tecnologias dominantes são as relacionadas com a eletricidade, produtos químicos e motores de combustão interna, as quais serviram de base para o desenvolvimento das tecnologias da quarta onda: aviação, corrida espacial, petroquímicos e eletrônicos. Por fim, a mais recente onda de inovação estabelecida se dá com a introdução dos computadores, criando a chamada era digital ou era das informações.

Assim como Hargroves e Smith (2013), Seebode, Jeanrenaud e Bessant (2012) consideram a sustentabilidade um sexto paradigma ou onda tecnológica em desenvolvimento, na qual o processo de inovação passa a se desenvolver, sendo caracterizado pela predominância de tecnologias voltadas para a produtividade radical de recursos, biomimética, química verde, ecologia industrial, energias renováveis e nanotecnologia verde, discutidos a seguir.

A produtividade radical de recursos, segundo Hart (2005), vai além da gestão responsável desses recursos na produção, consistindo no aumento da eficência dos recursos empregados, aumentando a quantidade de produtos com a utilização da mesma quantidade de recursos ou diminuir a quantidade de recursos necessários para a produção da mesma quantidade de produtos. Considerando que a economia depende criticamente dos recursos naturais, essa produtividade radical de recursos, portanto, é tida como a base da prosperidade econômica futura e da sobrevivência do planeta a longo prazo (WEISZÄCKER; AYRES, 2013).

Quanto à biomimética, Benyus (1997), biólogo e líder deste campo emergente, afirma que ela consiste na inovação inspirada pela natureza, ou seja, na produção de novos materiais ou estruturas com maior desempenho, resistência e tenacidade, copiando ou imitando elementos da natureza. Em outras palavras, é aprender com os projetos e soluções da natureza, aplicando-os na solução de problemas do dia a dia das pessoas. Nos últimos anos, conforme Lurie-Luke (2014), esta interpolação consciente de mecanismos e estruturas biológicas tem se difundido a partir de uma vasta gama de aplicações, como por exemplo: na arquitetura e design de interiores, pelo seu potencial na construção de soluções sustentáveis para problemas como iluminação, temperatura, eficência energética, durabilidade e produtividade (YELER, 2015); nos campos da química e da topografia de superfície (CHAPMAN et al., 2014); e no desenvolvimento da bioinformática (TRONCALE, 2014).

Outros elementos importantes para a sexta onda de inovação são a química verde e a nanotecnologia verde. A química verde é definida como o "planejamento de produtos e processos químicos no sentido de minimizar, ou mesmo eliminar, o uso de substâncias perigosas e a geração de poluentes como resíduos ou subprodutos" (GOES et al., 2013, p. 114). Diante dos atuais níveis de desenvolvimento e utilização de recursos naturais, a química verde se mostra fundamental para a proteção da saúde humana e do meio ambiente, desenvolvendo produtos e soluções em áreas como design de produtos, solventes benignos para o meio ambiente e matérias-primas renováveis (ANASTAS; KIRCHHOFF, 2002). Já a nanotecnologia verde, que consiste na criação de materiais, sistemas e dispositivos, úteis e funcionais, por meio da exploração de novos fenômenos e propriedades (físicas, químicas e biológicas), destaca-se pelo seu potencial para a sustentabilidade em diversas áreas, tais como saúde e medicina, energia e meio ambiente, transporte e exploração espacial, agricultura e alimentos (SOZER; KOKINI, 2012; YOUSAF; ALI, 2008). 
Aliada à química verde e à nanotecnologia verde, outro campo de rápido crescimento dentro da sexta onda de inovação é o da ecologia industrial, que analisa a indústria a partir do fluxo de materiais e gastos de energia, buscando a produção e consumo sustentável (LIFSET; GRAEDEL, 2015). Seu objetivo é, de acordo com Duchin e Levine (2014), identificar os impactos ambientais gerados pela produção industrial, rastreando os recursos utilizados e os resíduos. Tendo como principal ferramenta a avaliação do ciclo de vida, a ecologia industrial traz como benefícios a redução na entrada e o aumento na produtividade de recursos, além de reduzir resíduos, emissões e, consequentemente, o custo operacional da produção (DESPEISSE et al., 2012). Dessa maneira, estudos que demonstram a sua aplicação podem ser encontrados nos mais diversos campos, como por exemplo: produção de energia (GIURCO et al., 2011); setor de reciclagem (CORDER; GOLEV; GIURCO, 2015); abastecimento de água (COUSINS; NEWELL, 2015); indústria de alimentos e agricultura (SIMBOLI; TADDEO; MORGANTE, 2015); e metabolismo urbano (CONKE; FERREIRA, 2015).

Por fim, no que se refere à importância das energias renováveis para a nova onda de inovação, como as energias solar, éolica e da biomassa, Ming et al. (2013) relacionam sua necessidade com contextos de crescimento econômico acelerado, fontes de energia convencionais limitadas, alto consumo de energia e problemas graves de poluição. Neste sentido, as indústrias de geração de energia renovável são vistas como alternativas para outras fontes de energia, como mineração, processamento de petróleo e energia térmica (SONG et al., 2015). Não significa, contudo, afirmar que sua produção é isenta de gerar impactos ambientais. Ao contrário, impactos produzidos pela expansão de indústrias de energia renovável podem envolver espécies e até ecossistemas completos, exigindo avaliações constantes (BRAHAM et al., 2015).

De forma geral, cada uma dessas tecnologias e seus campos de atuação têm se desenvolvido como esforços na consolidação deste novo paradigma sustentável. Ressalta-se, contudo, que essa transformação deve se dar também no nível das ciências, introduzindo uma transdisciplinaridade que deve incorporar a sustentabilidade como meta coletiva (KLÄY; ZIMMERMANN; SCHNEIDER, 2015).

Além disso, devem ser consideradas correntes de pensamento relacionadas à área de convergência entre inovação e sustentabilidade, como o otimismo tecnológico e o paradoxo de Jevons, discutidas na próxima seção.

\section{OTIMISMO TECNOLÓGICO E PARADOXO DE JEVONS}

Um movimento que se dá em paralelo aos paradigmas tecnológicos e que não recebe grande atenção, apesar de se fazer presente em práticas modernas (KRIER; GILLETTE, 1985), é o chamado otimismo tecnológico, o qual, segundo Daily e Ehrlich (1992), consiste na crença de que o progresso técnico é capaz de levar a melhorias de eficiência, substituições de recursos e outras inovações. Evoluindo como uma resposta ao estudo malthusiano apresentado no relatório The limits to growth, do Clube de Roma (MEADOWS et al., 1972), o otimismo tecnológico é "a doutrina de que um número crescente de melhorias tecnológicas em áreas como a produção de alimentos, qualidade ambiental e energia irá sustentar a vida enquanto a população humana cresce” (BASIAGO, 1994, p. 17).

$\mathrm{O}$ argumento dos otimistas tecnológicos é de que problemas como poluição, esgotamento de recursos ou rápido crescimento populacional poderiam ser resolvidos apenas pela engenhosidade humana e o desenvolvimento de novas tecnologias (HUESEMANN, 2003), sem a necessidade de uma visão sistêmica desses problemas (KAMGA; YAZICI, 2014). Essas inovações seriam, portanto, capazes de promover transformações no estilo de vida da sociedade, garantindo a sua sustentabilidade. 
Contudo, Bella (1979) alerta para o fato de que essa visão otimisma desconsidera que tecnologia e ciência são empreendimentos humanos e, logo, possuem limitações sociais no seu desenvolvimento, como conhecimentos, valores, objetivos, etc. Além disso, também as próprias melhorias advindas com o desenvolvimento tecnológico não devem ser vistas em um caráter ilimitado, especialmente devido à chamada capacidade de carga do planeta, que se refere ao limite máximo de vida que um ecossistema pode suportar, a qual, mesmo que possa ser ampliada, não pode ser infinita, havendo sempre limites (DAILY; EHRLICH, 1992).

Tomando como exemplo ferramentas de ecoeficiência na produção industrial, Huesemann (2003) aponta que mesmo as melhores tecnologias humanas, no contexto das atividades industriais e econômicas, estão propensas a gerar consequências negativas para o meio ambiente que são inevitáveis. Outros estudos, em áreas como agricultura e produção de alimentos (HALL; HALL, 1993), degradação e desastres ambientais (PELLETIER, 2010; VRIES, 2011) e transporte urbano (MADDISON; WATTS, 2011), mostram como o otimismo tecnológico produz apenas soluções parciais. Otimismo tecnológico é, por conseguinte, apenas "um termo de arte, um artigo de fé e uma teoria política" (KRIER; GILLETTE, 1985, p. 405, tradução nossa).

Dessa maneira, Pollini (2009) considera que as práticas e tecnologias voltadas para a sustentabilidade devem se afastar de qualquer otimismo tecnológico, de modo a responder adequadamente aos desafios ambientais que permeiam a realidade do nosso tempo. Caso contrário, é grande a possibilidade de que a humanidade estará sempre aquém de desenvolver tecnologias necessárias para deter o ritmo de consumo e destruição dos recursos naturais do planeta, arriscando a sua sobrevivência (KARLSSON, 2014).

Além disso, conforme Polimeni e Polimeni (2006), apesar dos avanços tecnológicos mais eficientes, o consumo de recursos naturais tem aumentado consideravelmente nos últimos duzentos anos. Um cenário que pode interferir nos resultados benéficos esperados em torno de uma nova tecnologia é aquele que se reflete no chamado paradoxo de Jevons.

Apresentado inicialmente por Jevons (1865), considerado um dos pioneiros da economia ecológica (MISSEMER, 2012), esse paradoxo foi percebido em sua pesquisa acerca da utilização do carvão como fonte de energia no Reino Unido, na qual se constatou que o aumento na eficiência da queima de carvão ao invés de poupar este recurso, como era esperado, ampliou as suas possibilidades de uso econômico e, consequentemente, sua utilização. Logo, o paradoxo de Jevons vai se basear nessa observação de que uma melhoria na eficiência com a qual um recurso natural é utilizado frequentemente é associada a um aumento no consumo deste recurso (ALCOTT, 2005; YORK, 2006).

Assim, enquanto novas tecnologias são desenvolvidas a fim de reduzir os impactos das sociedades humanas sobre o meio ambiente, essas tecnologias também podem induzir a um nível mais elevado de exploração, tendo em vista aumentar a qualidade de vida dos indivíduos, mesmo que isso venha a reduzir os estoques de recursos. Um exemplo desse paradoxo é identificado por Ceddia et al. (2013) que, investigando o aumento das áreas de desmatamento na América do Sul, encontraram como principal causa a conversão de terras para a agricultura, motivada por tecnologias que possibilitam a sua intensificação.

Desse modo, reconhece-se que essa associação entre eficiência e o consumo total de um recurso se dá devido a outros fatores, como comportamentos de busca por lucro ou de expansão de produção, fazendo com que a eficiência de novas tecnologias não seja suficiente para a economia de recursos, devendo ser complementada com outras estratégias, como políticas de consumo e racionamento.

Ademais, deve-se destacar portanto a necessidade de reconhecer as influências que tanto 
o otimismo tecnológico como paradoxo de Jevons implicam no contexto do desenvolvimento de inovações sustentáveis, as quais serão exploradas na seção seguinte.

\section{INOVAÇÕES SUSTENTÁVEIS}

Segundo Merkel (1998), a inovação tecnológica oferece excelentes perspectivas para a sustentabilidade, sendo considerada por Kondoh et al. (2014) como um elemento indispensável das políticas desenvolvidas com este fim. Corroborando com essa afirmação, Raudsepp-Hearne et al. (2010) afirmam que o uso de tecnologias para melhorar a geração de serviços dos ecossistemas possui grande potencial, sendo chamadas neste contexto de tecnologias ou inovações sustentáveis. Estas últimas são definidas por Barbieri et al. (2010), Boons et al. (2013) e Cillo et al. (2019) como aquelas inovações que contribuem para a sustentabilidade, combinando valores e objetivos ambientais, econômicos e sociais. Em outras palavras, as inovações sustentáveis, que podem se apresentar como produtos, serviços, tecnologias e novos negócios ou modelos organizacionais, resultam de um processo de integração da sustentabilidade (ambiental, social, econômica) com os processos de inovação da empresa.

Foxon e Pearson (2008, p. 148, tradução nossa) definem as inovações sustentáveis como aquelas voltadas para "sistemas tecnológicos e processos institucionais mais sustentáveis, onde a utilização de recursos e produção de resíduos permanecem dentro de limites ambientais adequados e níveis socialmente aceitáveis de prosperidade econômica". O que esse tipo de inovação representa é uma relação direta entre os fenômenos da sustentabilidade e da inovação, na qual ambos estão voltados para mudanças em uma perspectiva de futuro: "Sustentabilidade diz respeito à preocupação com o bemestar futuro, e a inovação diz respeito à criação de novos produtos que podem gerar valor somente se eles se encaixam nessa perspectiva de futuro" (VERLOOP, 2004, p. 117, tradução nossa).

Conforme Hansen, Grosse-Dunker e Reichwald (2009), a importância das inovações sustentáveis se destaca nas mudanças dos próprios atributos utilizados para a mensuração de seu sucesso, sendo estes anteriormente puramente econômicos e, atualmente, envolvendo outros critérios como os da esfera ambiental: "A sustentabilidade coloca uma exigência normativa para que as inovações se tornem mais ambientalmente e socialmente benignas e, ao mesmo tempo, fornece uma nova fonte de inovações e vantagem competitiva" (HANSEN; GROSSE-DUNKER; REICHWALD, 2009, p. 685, tradução nossa). Dessa forma, essas inovações são nomeadas de inovações orientadas para a sustentabilidade (sustainability-oriented innovations ou sustainability-led innovations), demandando das organizações novas abordagens para o seu desenvolvimento (SEEBODE; JEANRENAUD; BESSANT, 2012).

Considerando os aspectos de incerteza e risco elevados presentes no processo de inovação tradicional, no caso das inovações sustentáveis, a complexidade do seu processo é aumentada (AKA, 2019). De acordo com Alkemade e Suurs (2012), não são apenas as forças de mercado que interferem na sua adoção, como a existência de tecnologias tradicionais ou sustentáveis concorrentes, mas externalidades relacionadas com a adoção e difusão dessas inovações como, por exemplo, níveis maiores de pureza no ar ou reservas de água mais limpas.

Além disso, como observado por Noppers et al. (2014), existem três tipos de atributos que interferem no processo de adoção de inovações sustentáveis pelos consumidores: atributos instrumentais, relacionados com os benefícios da tecnologia em si, como preço ou características físicas; atributos ambientais, que são os benefícios que a inovação traz para o meio ambiente ou para a sociedade, como redução de poluição ou consumo de energia; e atributos simbólicos, que são 
responsáveis por sinalizar características positivas para o consumidor, como autoidentidade ou status social. Destes três tipos de atributos, são os atributos simbólicos e ambientais os mais importantes na adoção de inovações sustentáveis.

De forma geral, resumem-se em três os principais desafios que as inovações sustentáveis têm que superar: atender as expectativas gerais da sociedade; criar uma distribuição equitativa de valor ao longo de sua cadeia de valor; e, por fim, se encaixar dentro da capacidade de carga dos seus ecossistemas de suporte (VERLOOP, 2004). Desta maneira, a fim de ser bem sucedidas, as inovações sustentáveis requerem tecnologias e modelos de negócios benignos, que atendam às necessidades de todos os stakeholders e provoquem mudanças em sintonia com as necessidades da sociedade.

Tais inovações, além de carregar os elementos de sustentabilidade que lhe são exigidos, devem manter características de sucesso para o seu usuário, sendo as principais, segundo Hynds (2013): velocidade, ao minimizar o tempo gasto com determinada tarefa; variação, ao produzir resultados diferentes dos anteriores; e eficiência, a partir da utilização de menos recursos para o alcance de mais resultados. A sustentabilidade, portanto, seria uma quarta característica que adicionaria valor para a inovação.

Ao contrário das ecoinovações, cujo foco está na busca da ecoeficiência (intercessão entre as dimensões ambiental e econômica), as inovações sustentáveis e as organizações inovadoras sustentáveis devem ir além deste contexto, focando também nos princípios de equidade (dimensões social e econômica) e habilidade (dimensões ambiental e social), já que a sustentabilidade se preocupa tanto com o bem-estar social quanto com os impactos ecológicos (BARBIERI et al., 2010; GOBBLE, 2012).

Inovações sustentáveis não apenas tornam mais verde as empresas, como também aumentam a sua competitividade. Sendo assim, os impactos das inovações sustentáveis podem ser observados em três níveis distintos: no nível micro, em um produto, serviço ou processo de determinada empresa; no nível médio, em um setor, cadeia de suprimentos, região ou sistema de produto; e no nível macro, que pode ser toda a economia de um país, um bloco econômico ou mesmo todo o planeta (CASTIAUX, 2012).

Para o seu desenvolvimento, Boons et al. (2013) asseveram a necessidade de um modelo de negócios que permita às empresas a construção de uma estrutura holística que avalie todo o contexto dessas inovações, identificando relações entre a empresa e seu sistema mais amplo de produção e consumo. Ademais, como observado por Aka (2019), os papéis de atores como produtores, avaliadores, investidores, reguladores, promotores, certificadores e influenciadores da inovação sustentável são fundamentais. Assim, as empresas são movidas a criar propostas de valor, organizar sua cadeia de valor e estabelecer um modelo financeiro que possibilite o sucesso de inovações sustentáveis no mercado, respeitando toda a complexidade e relações inerentes ao seu processo de adoção.

Em complemento, Barbieri et al. (2010) enfatizam ainda a abordagem da gestão estratégica de nicho (strategic niche management), quando um produto com novas tecnologias é introduzido em pequenos mercados para avaliação, facilitando a introdução dessas inovações sustentáveis com a utilização de nichos de negócios para a sua experimentação, tendo em vista a difusão posterior na sociedade. Essa abordagem é explorada, por exemplo, no estudo de Smith et al. (2014), realizado no contexto da geração de energia solar fotovoltática no Reino Unido, que aponta a importância dessa estratégia na capacitação e fortalecimento de novas tecnologias para o mercado.

Além disso, mais do que a mera introdução de uma nova tecnologia voltada para a sustentabilidade, é preciso que sejam criadas políticas de incentivo à sua utilização, garantindo a aceitação e difusão de tais tecnologias entre os cidadãos (VAN DER WERFF; PERLAVICIUTE; MUIÑOS, 2016). De acordo com Foxon e Pearson (2008), esses princípios de criação de políticas de inovação sustentável 
destacam a importância da construção de relações evolutivas entre políticas ambientais e de inovação, considerando ainda a complexidade e as interações entre os sistemas de inovação e os sistemas de decisão política de forma a promover uma transição para a sustentabilidade.

Neste contexto, destacam-se como agentes fundamentais neste processo de transição para a sustentabilidade, mediante o desenvolvimento de inovações sustentáveis, as organizações inovadoras sustentáveis, cujos aspectos serão melhor explorados na seção seguinte.

\section{ORGANIZAÇÕES INOVADORAS E SUSTENTÁVEIS}

Organizações inovadoras, segundo Veer (2004), são aquelas que têm capacidade de aplicar novos conhecimentos mais rápido do que seus concorrentes, por meio da promoção de inovações, surpreendendo seus clientes com novas experiências de consumo. São empresas que geralmente operam dentro de ambientes tecnológicos e legais turbulentos, onde são confrontadas por mudanças constantes e inesperadas (SHAREEF, 1991).

Conforme West e Sacramento (2012), suas principais características são: crença firme e partilhada entre os membros acerca dos objetivos da organização; alto nível de interação, discussão e debate construtivo entre os membros da organização; confiança, cooperação e uma sensação de segurança nas relações interpessoais e intergrupais; administração positiva e aberta às novas ideias; incentivos e recursos para a inovação; e criatividade como fator-chave para sobrevivência e sucesso organizacional. Em contrapartida, empresas cujo clima organizacional é caracterizado pela desconfiança, falta de comunicação, antipatias pessoais, autonomia individual limitada e objetivos pouco claros, são espaços onde o processo de inovação tende a ser extremamente deficiente (WEST, 2001).

Analisando um quadro mais amplo de componentes relacionados com o sucesso dessas organizações inovadoras, Tidd e Bessant (2018) destacam que as organizações inovadoras não são organizações que se sobressaem apenas pela sua estrutura, mas muitos outros aspectos, como liderança, trabalho em equipe e aprendizagem organizacional. Neste sentido, é preciso compreender todo o processo de gestão da inovação neste tipo de empresas.

De acordo com Dodgson, Gann e Salter (2008), são cinco os modelos de inovação que essas organizações podem utilizar, de forma a orientar o seu processo de inovação para a estratégia corporativa. O primeiro modelo do processo de inovação é caracterizado pelo foco no desenvolvimento de pesquisa e envio de novas tecnologias para o mercado, em uma dinâmica linear, que passa pelos processos de desenvolvimento, manufatura e marketing da empresa. Quanto ao segundo modelo, este mantém essa perspectiva linear do processo de inovação, mas parte da demanda do mercado, com as atividades de pesquisa tecnológica voltadas para o atendimento das necessidades dos clientes. Considerando a vinculação que deve existir entre as pesquisas e o mercado, o terceiro modelo destaca essa necessidade de interação, comfeedbacks constantes entre os dois extremos do processo de inovação. Já o quarto modelo destaca a importância de agentes como fornecedores e consumidores em um processo colaborativo de inovação. Finalmente, o quinto e último modelo seria o mais completo, com a total integração do processo de inovação com a estratégia organizacional e com os demais agentes de inovação.

De modo geral, considera-se que as organizações inovadoras podem trabalhar com elementos de diferentes modelos no seu processo de inovação dependendo, por exemplo, do setor ou grau de complexidade da tecnologia desenvolvida. Inovações mais simples, de um tipo incremental, podem ser desenvolvidas com modelos mais lineares, impulsionados pela pesquisa ou pelo mercado, enquanto 
inovações mais radicais obter um sucesso de mercado maior quanto desenvolvidas em processos que integrem a estratégia organizacional e outros agentes de inovação, à semelhança do quinto modelo. O importante, neste sentido, é adequar estratégia, mercado, recursos e capacidades para a inovação.

Ademais, é importante ressaltar uma distinção neste contexto das organizações inovadoras, que é a existência de organizações mais voltadas para a geração de inovações e organizações mais voltadas para a adoção de inovações (DAMANPOUR; WISCHNEVSKY, 2006). Considerando que os processos de desenvolvimento e adoção de inovações são distintos, as organizações inovadoras, mesmo que adotem inovações de outras empresas nos seus processos e produtos, são aquelas que tem como estratégia primária a geração de novas tecnologias. Além disso, essas organizações incentivam a criatividade e a inovação em vários níveis (individual, de grupo e organizacional), reconhecendo esse processo como crítico para o sucesso organizacional (MUMFORD; HESTER; ROBLEDO, 2012).

Localizadas na área de convergência entre as organizações inovadoras e as organizações sustentáveis, já discutidas anteriormente na seção sobre a sustentabilidade, estão as organizações inovadoras sustentáveis. Segundo Barbieri et al. (2010), essas organizações são aquelas que buscam a inovação respeitando as dimensões do triple bottom line: na dimensão social, preocupando-se com os impactos sociais das inovações tanto para os indivíduos dentro da organização quanto para a sociedade, como desemprego, exclusão social, pobreza e diversidade organizacional; na dimensão ambiental, preocupando-se com os impactos que a utilização dos recursos naturais e a emissão de poluentes e resíduos geram no meio ambiente; e na dimensão econômica, preocupando-se com a chamada eficiência econômica, essencial para a manutenção da saúde financeira da organização e sua perpetuação.

Nessa busca de equilíbrio entre essas três dimensões, as organizações inovadoras sustentáveis buscam agregar valor ambiental e social para o ambiente externo, a partir do seu core business (KONDOH et al., 2014). Sua importância, como afirmado por Nakata e Viswanathan (2012), se destaca junto aos mercados na base da pirâmide, mediante a geração de empreendimentos de impacto econômico e social, baseados em inovações sustentáveis que atendam às suas necessidades.

\section{CONSIDERAÇÕES FINAIS}

Partindo da influência que os diversos paradigmas e correntes de pensamento exercem sobre a produção de novas tecnologias, há que se reconhecer que as inovações não são independentes do seu contexto social, com o qual interagem tanto nas fases de sua concepção e produção como no que diz respeito às consequências que produz. Tal percepção se mostrou na discussão inicial deste ensaio em torno dos paradigmas da sustentabilidade, e as influências do otimismo tecnológico e do paradoxo de Jevons neste contexto.

De fato, o relatório Nosso Futuro Comum da WCED (1987), por exemplo, já apontava o desenvolvimento tecnológico como um dos elementos que interfere diretamente nos limites do desenvolvimento e da sustentabilidade. Sendo assim, as inovações se apresentam como essenciais para o atendimento de metas sustentáveis, seja promovendo o bem-estar das economias, com mudanças nas estratégias e rotinas das empresas, ou a saúde dos diversos ecossistemas ao redor do mundo, superando problemas como a poluição e degradação ambiental.

Reconhecendo que grande parte da difusão inicial do paradigma sustentável na segunda metade do século XX se deu exatamente em decorrência dos problemas ambientais causados por inovações, é preciso cada vez mais que as inovações gerem resultados positivos em todas as dimensões da 
sustentabilidade. Diante da preocupação comum aos envolvidos nos debates em torno da sustentabilidade, que é a de que os recursos fixos ou finitos do ecossistema natural não sejam capazes de suportar a dinâmica de crescimento das atividades humanas, denota-se que inovações, quando concebidas e geridas em harmonia com o pensamento sustentável, podem ser capazes de inverter as tendências atuais de um futuro insustentável. Para isso, os desafios destas inovações concentram-se basicamente em utilizar de novas fontes ou alternativas de recursos, principalmente se um recurso natural está se esgotando, ou auxiliar na utilização e reutilização desses recursos de maneira mais eficiente.

De forma complementar, assim como as inovações se apresentam como elemento necessário para a sustentabilidade, também a sustentabilidade se coloca como um paradigma essencial no processo de desenvolvimento de inovações, como destacado nas discussões em torno das inovações sustentáveis e das organizações inovadoras e sustentáveis. Frente à crescente demanda por serviços dos ecossistemas, o desenvolvimento e a difusão de inovações destinadas a aumentar a eficiência do uso de seus recursos ou com vistas a reduzir os impactos ocasionados pelas atividades humanas são essenciais.

Ademais, espera-se que a partir das discussões lançadas neste ensaio, que teve como objetivo analisar os caminhos e desafios de inovações e organizações rumo à sustentabilidade, fortaleçam-se os esforços de pesquisa nesta área, com a realização de novas investigações que possam aprofundar e apreciar temas ainda pouco explorados neste campo, como as características de inovações advindas do paradigma sustentável (produtividade radical de recursos, biomimética, química verde, ecologia industrial, energias renováveis e nanotecnologia verde), as trajetórias de desenvolvimento de inovações sustentáveis ou de aprendizagem em organizações inovadoras e sustentáveis.

Desse modo, o que se incita é o fortalecimento desta evolução de pesquisas, discussões e resultados que cada vez mais considerem inovação e sustentabilidade como dois lados de uma mesma moeda ou, quem sabe até fenômenos presentes em um mesmo lado dessa moeda chamada desenvolvimento sustentável que, dada a sua complexidade, pode apresentar ainda tantos outros lados.

\section{REFERÊNCIAS}

AKA, K. G. Actor-network theory to understand, track and succeed in a sustainable innovation development process. Journal of Cleaner Production, v. 225, p. 524-540, 2019. doi: 10.1016/j. jclepro.2019.03.351.

ALCOTT, B. Jevons' paradox. Ecological Economics, v. 54, n. 1, p. 9-21, 2005. doi: 10.1016/j. ecolecon.2005.03.020.

ALKEMADE, F.; SUURS, R. A. A. Patterns of expectations for emerging sustainable technologies. Technological Forecasting and Social Change, v. 79, n. 3, p. 448-456, 2012. doi: 10.1016/j. techfore.2011.08.014.

ANASTAS, P. T.; KIRCHHOFF, M. M. Origins, current status, and future challenges of green chemistry. Accounts of Chemical Research, v. 35, n. 9, p. 686-694, 2002. doi: 10.1021/ar010065m.

ANTHONY JR., B. Green information system integration for environmental performance in organizations: An extension of belief-action-outcome framework and natural resource-based view theory. Benchmarking: An International Journal, v. 26, n. 3, p. 1033-1062, 2019. doi: 10.1108/BIJ05-2018-0142. 
BARBIERI, J. C. et al. Inovação e sustentabilidade: novos modelos e proposições. Rev. adm. empres., v. 50, n. 2, p. 146-154, 2010.

BASIAGO, A. D. The limits of technological optimism. Environmentalist, v. 14, n. 1, p. 17-22, 1994. doi: 10.1007/BF01902656.

BELLA, D. A. Technological constraints on technological optimism. Technological Forecasting and Social Change, v. 14, n. 1, p. 15-26, 1979. doi: 10.1016/0040-1625(79)90053-2.

BENYUS, J. M. Biomimicry: innovation inspired by nature. New York: Harper Collins Publishers, 1997.

BOONS, F. et al. Sustainable innovation, business models and economic performance: an overview. Journal of Cleaner Production, v. 45, p. 1-8, 2013. doi: 10.1016/j.jclepro.2012.08.013.

BRAHAM, M. et al. Home in the heat: dramatic seasonal variation in home range of desert golden eagles informs management for renewable energy development. Biological Conservation, v. 186, p. 225-232, 2015. doi: 10.1016/j.biocon.2015.03.020.

CASTIAUX, A. Developing dynamic capabilities to meet sustainable development challenges. International Journal of Innovation Management, v. 16, n. 6, p. 1-16, 2012. doi: 10.1142/ S1363919612400130.

CEDDIA, M. G. et al. Sustainable agricultural intensification or Jevons paradox? The role of public governance in tropical South America. Global Environmental Change, v. 23, n. 5, p. 1052-1063, 2013. doi: 10.1016/j.gloenvcha.2013.07.005.

CHAPMAN, J. et al. Bioinspired synthetic macroalgae: examples from nature for antifouling applications. International Biodeterioration \& Biodegradation, v. 86, n. 1, p. 6-13, 2014. doi: 10.1016/j.ibiod.2013.03.036.

CILLO, V. et al. Understanding sustainable innovation: A systematic literature review. Corporate Social Responsibility and Environmental Management, v. 26, n. 5, p. 1012-1025, 2019. doi: 10.1002/csr.1783.

CONKE, L. S.; FERREIRA, T. L. Urban metabolism: measuring the city's contribution to sustainable development. Environmental Pollution, v. 202, p. 146-152, 2015. doi: 10.1016/j. envpol.2015.03.027.

CORDER, G. D.; GOLEV, A.; GIURCO, D. "Wealth from metal waste": translating global knowledge on industrial ecology to metals recycling in Australia. Minerals Engineering, v. 76, p. 2-9, 2015. doi: 10.1016/j.mineng.2014.11.004.

COUSINS, J. J.; NEWELL, J. P. A political-industrial ecology of water supply infrastructure for Los Angeles. Geoforum, v. 58, p. 38-50, 2015. doi: 10.1016/j.geoforum.2014.10.011.

DAILY, G. C.; EHRLICH, P. R. Population, sustainability, and earth's carrying capacity: a framework for estimating population sizes and lifestyles that could be sustained without undermining future generations. BioScience, v. 42, n. 10, p. 761-771, 1992.

DAMANPOUR, F.; WISCHNEVSKY, J. D. Research on innovation in organizations: distinguishing innovation-generating from innovation-adopting organizations. Journal of Engineering and Technology Management, v. 23, n. 4, p. 269-291, 2006. doi: 10.1016/j.jengtecman.2006.08.002. 
DESPEISSE, M. et al. Industrial ecology at factory level - a conceptual model. Journal of Cleaner Production, v. 31, p. 30-39, 2012. doi: 10.1016/j.jclepro.2012.02.027.

DIAS, R. C.; SEIXAS, P. C. Regional models of sustainability governance: An analysis of the first integrated strategies for territorial development in Portugal. Revista Portuguesa de Estudos Regionais, v. 48, p. 5-16, 2018.

DODGSON, M.; GANN, D.; SALTER, A. The management of technological innovation: strategy and practice. New York: Oxford University Press, 2008.

DOSI, G. Sources, procedures and microeconomic effects of innovation. Journal of Economic Literature, v. 26, n. 3, p. 1120-1171, 1988.

DOSI, G. Technological paradigms and technological trajectories: a suggested interpretation of the determinants and directions of technical change. Research Policy, v. 11, n. 3, p. 147-162, 1982.

DUCHIN, F.; LEVINE, S. H. Industrial Ecology. Reference Module in Earth Systems and Environmental Sciences, p. 1968-1975, 2014. doi: 10.1016/B978-0-12-409548-9.09407-0.

DUMITRIU, D. et al. A perspective over modern SMES: managing brand equity, growth and sustainability through digital marketing tools and techniques. Sustainability, v. 11, n. 7, p. 2111, 2019. doi: $10.3390 /$ su11072111

FALQI, I. I.; AHMED, M.; MALLICK, J. Siliceous concrete materials management for sustainability using Fuzzy-TOPSIS Approach. Applied Sciences, v. 9, n. 17, p. 3457, 2019. doi: 10.3390/app9173457.

FOXON, T.; PEARSON, P. Overcoming barriers to innovation and diffusion of cleaner technologies: some features of a sustainable innovation policy regime. Journal of Cleaner Production, v. 16, n. 1, p. 148-161, 2008. doi: 10.1016/j.jclepro.2007.10.011.

FREEMAN, C.; PEREZ, C. Structural crises of adjustment, business cycles and investment behavior. In: DOSI, G., et al. Technical change and economic theory. London: Pinter, 1988.

GIL, J. et al. Reconciling global sustainability targets and local action for food production and climate change mitigation. Global Environmental Change, v. 59, p. 101983, 2019. doi: 10.1016/j. gloenvcha.2019.101983.

GIURCO, D. et al. Backcasting energy futures using industrial ecology. Technological Forecasting and Social Change, v. 78, n. 5, p. 797-818, 2011. doi: 10.1016/j.techfore.2010.09.004.

GOBBLE, M. M. Innovation and Sustainability. Research-Technology Management, v. 55, n. 5, p. 64-66, 2012. doi: 10.5437/08956308X5505005.

GOES, L. F. et al. Aspectos do conhecimento pedagógico do conteúdo de química verde em professores universitários de química. Educación Química, v. 24, n. 1, p. 113-123, 2013. doi: 10.1016/S0187-893X(13)72504-7.

HALL, C. A. S.; HALL, M. H. P. The efficiency of land and energy use in tropical economies and agriculture. Agriculture, Ecosystems \& Environment, v. 46, p. 1-30, 1993. doi: 10.1016/01678809(93)90010-M. 
HALL, J.; VREDENBURG, H. The challenges of innovating for sustainable development. MIT Sloan Management Review, v. 45, n. 1, p. 61-68, 2003.

HANSEN, E. G.; GROSSE-DUNKER, F.; REICHWALD, R. Sustainability innovation cube - a framework to evaluate sustainability-oriented innovations. International Journal of Innovation Management, v. 13, n. 4, p. 683-713, 2009.

HARGROVES, K. C.; SMITH, M. H. The natural advantage of nations: business opportunities, innovation and governance in the 21st century. London: Earthscan, 2013.

HART, S. L. Innovation, creative destruction and sustainability. Research-Technology Management, v. 48, n. 5, p. 21-27, 2005.

HUESEMANN, M. H. The limits of technological solutions to sustainable development. Clean Technologies and Environmental Policy, v. 5, n. 1, p. 21-34, 2003. doi: 10.1007/s10098-002-0173-8. HYNDS, E. J. Viewing innovation through the sustainability lens. Research-Technology Management, v. 56, n. 2, p. 10-12, 2013.

JACINTO, R. C. et al. Use of waste from the production chain of pinion for the production of pellets for energy generation. Floresta, v. 47, n. 3, p. 353-363, 2017. doi: 10.5380/rf.v47i1.52080.

JEVONS, W. S. The coal question: an inquiry concerning the progress of the nation, and the probable exhaustion of our coal-mines. London: Macmillan and Co., 1865.

KALISH, D. et al. Integrating sustainability into new product development: available tools and frameworks can help companies ensure that sustainability is embedded as a fundamental building block of new product development. Research-Technology Management, v. 61, n. 2, p. 37-46, 2018. doi: 10.1080/08956308.2018.1421379.

KAMGA, C.; YAZICI, M. A. Achieving environmental sustainability beyond technological improvements: potential role of high-speed rail in the United States of America. Transportation Research, v. 31, p. 148-164, 2014. doi: 10.1016/j.trd.2014.06.011.

KARLSSON, R. Theorizing sustainability in a post-Concorde world. Technology in Society, v. 39, p. 1-9, 2014. doi: 10.1016/j.techsoc.2014.05.004.

KLÄY, A.; ZIMMERMANN, A. B.; SCHNEIDER, F. Rethinking science for sustainable development: reflexive interaction for a paradigm transformation. Futures, v. 65, p. 72-85, 2015. doi: 10.1016/j.futures.2014.10.012.

KONDOH, S. et al. Toward a sustainable business design: a survey. Procedia CIRP, v. 15, p. $367-$ 372, 2014. doi: 10.1016/j.procir.2014.06.094.

KRIER, J. E.; GILLETTE, C. P. The un-easy case for technological optimism. Michigan Law Review, v. 84, n. 3, p. 405-429, 1985.

LAVARELLO, P. Convergencia de paradigmas biotecnológicos y estrategias de los grupos líderes mundiales. Problemas del Desarrollo, v. 45, p. 9-35, 2014. doi: 10.1016/S0301-7036(14)70861-2.

LAZZAROTTI, F. et al. Tecnologias ambientais e os impactos no desempenho econômicofinanceiro: o caso da Celulose Irani S/A. Contextus - Revista Contemporânea de Economia e Gestão, v. 12, n. 1, p. 56-80, 2014. doi: 10.19094/contextus.v12i1.32171. 
LHERMIE, G. et al. Tradeoffs between resistance to antimicrobials in public health and their use in agriculture: Moving towards sustainability assessment. Ecological Economics, v. 166, p. 106427, 2019. doi: 10.1016/j.ecolecon.2019.106427.

LIFSET, R.; GRAEDEL, T. E. Industrial ecology. In: WRIGHT, J. D. International Encyclopedia of the Social \& Behavioral Sciences. Amsterdam, Netherlands: Elsevier, 2015. doi: 10.1016/B978-008-097086-8.91023-7.

LURIE-LUKE, E. Product and technology innovation: what can biomimicry inspire? Biotechnology Advances, v. 32, n. 8, p. 1494-1505, 2014. doi: 10.1016/j.biotechadv.2014.10.002.

MADDISON, J.; WATTS, R. The technological fix as a frame in media debates about tailpipe emissions. Technology in Society, v. 33, p. 294-303, 2011. doi: 10.1016/j.techsoc.2011.10.003.

MEADOWS, D. H. et al. The limits to growth. New York. 1972.

MERKEL, A. The role of science in sustainable development. Science, v. 281, n. 5375, p. 336-337, 1998. doi: 10.1126/science.281.5375.336.

MING, Z. et al. Overall review of renewable energy tariff policy in China: Evolution,

implementation, problems and countermeasures. Renewable and Sustainable Energy Reviews, v. 25, p. 260-271, 2013. doi: 10.1016/j.rser.2013.04.026.

MISSEMER, A. William Stanley Jevons' The Coal Question (1865), beyond the rebound effect. Ecological Economics, v. 82, p. 97-103, 2012. doi: 10.1016/j.ecolecon.2012.07.010.

MUMFORD, M. D.; HESTER, K. S.; ROBLEDO, I. C. Creativity in organizations: importance and approaches. In: MUMFORD, M. D. Handbook of Organizational Creativity. London, UK: Academic Press, 2012.

NAKATA, C.; VISWANATHAN, M. From impactful research to sustainable innovations for subsistence marketplaces. Journal of Business Research, v. 12, p. 1655-1657, 2012. doi: 10.1016/j. jbusres.2012.02.005.

NOPPERS, E. H. et al. The adoption of sustainable innovations: driven by symbolic and environmental motives. Global Environmental Change, v. 25, p. 52-62, 2014. doi: 10.1016/j. gloenvcha.2014.01.012.

PELLETIER, N. Of laws and limits: An ecological economic perspective on redressing the failure of contemporary global environmental governance. Global Environmental Change, v. 20, n. 2, p. 220-228, 2010. doi: 10.1016/j.gloenvcha.2009.12.006.

POLLINI, J. Agroforestry and the search for alternatives to slash-and-burn cultivation: from technological optimism to a political economy of deforestation. Agriculture, Ecosystems \& Environment, v. 133, p. 48-60, 2009. doi: 10.1016/j.agee.2009.05.002.

RAUDSEPP-HEARNE, C. et al. Untangling the environmentalist's paradox: why is human wellbeing increasing as ecosystem services degrade? BioScience, v. 60, n. 8, p. 576-589, 2010. doi: 10.1525/bio.2010.60.8.4.

SEEBODE, D.; JEANRENAUD, S.; BESSANT, J. Managing innovation for sustainability. $R \& D$ Management, v. 42, n. 3, p. 195-206, 2012. 
SHAREEF, R. Ecovision: a leadership theory for innovative organizations. Organizational Dynamics, v. 20, n. 1, p. 50-62, 1991. doi: 10.1016/0090-2616(91)90082-K.

SIMBOLI, A.; TADDEO, R.; MORGANTE, A. The potential of industrial ecology in agri-food clusters (AFCs): a case study based on valorisation of auxiliary materials. Ecological Economics, v. 111, p. 65-75, 2015. doi: 10.1016/j.ecolecon.2015.01.005.

SMITH, A. et al. Spaces for sustainable innovation: solar photovoltaic electricity in the UK. Technological Forecasting and Social Change, v. 81, p. 115-130, 2014. doi: 10.1016/j. techfore.2013.02.001.

SONG, J. et al. Introducing renewable energy and industrial restructuring to reduce GHG emission: application of a dynamic simulation model. Energy Conversion and Management, v. 96, p. 625-636, 2015. doi: 10.1016/j.enconman.2015.03.024.

SOZER, N.; KOKINI, J. L. The applications of nanotechnology. In: PICÓ, Y. Chemical analysis of food: techniques and applications. Waltham, MA: Academic Press, 2012. doi: 10.1016/B978-0-12384862-8.00006-6.

TIDD, J.; BESSANT, J. Managing innovation: integrating technological, market and organizational change. $6^{\text {th }}$ ed. New York: John Wiley \& Sons, 2018.

TORRES, S.; ALVES, S.; VIDAL, F. Os parques tecnológicos como agentes imobiliários na América Latina. Contextus - Revista Contemporânea de Economia e Gestão, v. 15, n. 1, 2017. doi: 10.19094/contextus.v15i1.883

TRONCALE, L. Sysinformatics \& systems mimicry: new fields emerging from a "science" of systems processes engineering. Procedia Computer Science, v. 28, p. 663-671, 2014. doi: 10.1016/j. procs.2014.03.080.

VAN DER WERFF, E.; PERLAVICIUTE, G.; MUIÑOS, G. Current developments in environmental psychology: topics and researchers. Psyecology, v. 7, n. 3, p. 229-235, 2016. doi: 10.1080/21711976.2016.1251105

VEER, J. V. Foreword: a sustainable planet needs sustainable innovation. In: VERLOOP, J. Insight in innovation: managing innovation by understanding the laws of innovation. Amsterdam, Netherlands: Elsevier, 2004.

VERLOOP, J. Sustainable innovation. Amsterdam, Netherlands: Elsevier, 2004.

VOLLENBROEK, F. A. Sustainable development and the challenge of innovation. Journal of Cleaner Production, v. 10, n. 3, p. 215-223, 2002. doi: 10.1016/S0959-6526(01)00048-8.

VRIES, D. H. Temporal vulnerability in hazardscapes: flood memory-networks and referentiality along the North Carolina Neuse River (USA). Global Environmental Change, v. 21, n. 1, p. 154164, 2011. doi: 10.1016/j.gloenvcha.2010.09.006.

WEISZÄCKER, E. U.; AYRES, R. U. Boosting resource productivity: creating ping-pong dynamics between resource productivity and resource prices. Environmental Innovation and Societal Transitions, v. 9, p. 48-55, 2013. doi: 10.1016/j.eist.2013.09.001. 
WEST, M. A. Management of creativity and innovation in organizations. In: SMELSER, N. J.; BALTES, P. B. International Encyclopedia of the Social \& Behavioral Sciences. Amsterdam, Netherlands: Elsevier, 2001. doi: 10.1016/B0-08-043076-7/01418-2.

WEST, M. A.; SACRAMENTO, C. A. Creativity and innovation: the role of team and organizational climate. In: MUMFORD, M. D. Handbook of organizational creativity. London, UK: Academic Press, 2012.

WICKI, S.; HANSEN, E. G. Green technology innovation: Anatomy of exploration processes from a learning perspective. Business Strategy and the Environment, v. 28, n. 6, p. 970-988, 2019. doi: 10.1002/bse.2295.

WORLD COMMISSION ON ENVIRONMENT AND DEVELOPMENT - WCED. Our Common Future. New York: WCED, 1987.

YELER, G. M. Creating nature awareness in design education. Procedia - Social and Behavioral Sciences, v. 174, p. 406-413, 2015. doi: 10.1016/j.sbspro.2015.01.682.

YORK, R. Ecological paradoxes: William Stanley Jevons and the paperless office. Human Ecology Review, v. 13, n. 2, p. 143-147, 2006.

YOUSAF, A. S.; ALI, S. Why nanoscience and nanotechnology? What is there for us. Journal of Faculty of Engineering \& Technology, v. 5, p. 11-20, 2008. 